\title{
Hypervascular Liver Metastasis from Hypovascular Ductal Cell Carcinoma of the Pancreas
}

\author{
Ayako Noda, Koji Inaba, Sonoko Sakuragi, Toshihisa Moriguchi, Toshihito Tanahashi, Toshiyuki Komaki, \\ Hiroyuki Kimura, Koichi Hirakawa, Naoki Teramae, Susumu Fukui, Yoichi Nishimoto and Keizo Kagawa
}

\begin{abstract}
In a case of hypervascular metastatic liver tumor, the vascularity of primary focus, pancreatic carcinoma was hypovascular. Based on the imaging findings, we thought before the operation that the two lesions were double cancers. Histological examination showed that the stromal volume of metastatic tumorous tissue was richer than that of the primary focus. It was suggested that the difference in the stromal volume was related to the difference of the vascularity. Some foctors originating in stromal cells might be involved in angiogenesis.
\end{abstract}

(Internal Medicine 40: 227-231, 2001)

Key words: vascularity, angiogenesis, stromal cell

\section{Introduction}

The vascularity of metastatic tumor is usually similar to that of the primary focus. Adenocarcinoma in the digestive system often occurs in liver metastasis; the vascularity is generally hypovascular.

We describe a very rare case of hypervascular metastatic liver tumor in whom the vascularity of the primary focus, pancreatic ductal cell carcinoma, was hypovascular.

\section{Case Report}

A 61-year-old man visited our hospital on October 1, 1998, because of left lateral abdominal pain. A large hepatic tumor was detected on screening ultrasonography, therefore he was admitted to our hospital for close examination.

On physical examination on admission, he had no abnormarity.

He had normal hematological findings, and no significant biochemical abnormality. Tumor markers such as CEA, CA199, DUPAN-2 and SPan-1, were not elevated. HBs antigen and
HCV antibody were negative (Table 1).

Abdominal ultrasonography detected a hyperechoic tumor of about $70 \mathrm{~mm}$ in diameter at the lateral segment of the liver and a hypoechoic tumor of about $30 \mathrm{~mm}$ in diameter at the tail of pancreas (Fig. 1). On plain computed tomographic (CT) scan these were low-density masses. The rapid enhancement effect of the hepatic tumor was observed during dynamic contrast CT scan. On the other hand, the enhancement effect of the pancreatic tumor was poor (Fig. 2). T1-weighted magnetic resonance imaging (MRI) showed the hepatic tumor as a low intensity mass. T2-weighted MRI showed it as a very high intensity mass. Both T1 and T2 weighted MRI showed the pancreatic tumor as a low intensity mass (Fig. 3). The endoscopic

Table 1. Laboratory Data on Admission

\begin{tabular}{lrlr}
\hline WBC & $6,100 / \mu l$ & T.P. & $7.1 \mathrm{~g} / \mathrm{dl}$ \\
RBC & $435 \times 10^{4} / \mu \mathrm{l}$ & AMY & $76 \mathrm{IU} / l$ \\
$\mathrm{Hb}$ & $12.5 \mathrm{~g} / \mathrm{dl}$ & BUN & $11 / \mathrm{dl}$ \\
$\mathrm{Ht}$ & $44.6 \%$ & CRE & $0.7 / \mathrm{dl}$ \\
$\mathrm{Plt}$ & $17.2 \times 10^{4} / \mu \mathrm{l}$ & $\mathrm{Na}$ & $140 \mathrm{mEq} / l$ \\
$\mathrm{CRP}$ & $0.2 / \mathrm{dl}$ & $\mathrm{K}$ & $3.8 \mathrm{mEq} / l$ \\
$\mathrm{LDH}$ & $340 \mathrm{IU} / l$ & $\mathrm{Cl}$ & $108 \mathrm{mEq} / l$ \\
GOT & $15 \mathrm{IU} / l$ & ICGR15 & $5.1 \%$ \\
GPT & $17 \mathrm{IU} / l$ & AFP & $8.0 \mathrm{ng} / \mathrm{ml}$ \\
ALP & $203 \mathrm{IU} / l$ & CEA & $1.1 \mathrm{ng} / \mathrm{ml}$ \\
$\gamma-$ GTP & $62 \mathrm{IU} / l$ & CA19-9 & $<2.0 \mathrm{U} / \mathrm{ml}$ \\
T.bil & $0.9 \mathrm{mg} / \mathrm{dl}$ & DUPAN-2 & $<25 \mathrm{U} / \mathrm{ml}$ \\
Ch.E. & $168 \mathrm{IU} / l$ & SPan- 1 & $<3.0 \mathrm{U} / \mathrm{ml}$ \\
& & HBsAg & $(-)$ \\
& & HCVAb & $(-)$ \\
\hline
\end{tabular}

WBC: white blood cell, RBC: red blood cell, Hb: hemoglobin, Ht: hematocrit, Plt: platelet, CRP: C-reactive protain, LDH: lactate dehydrogenase, AST: asparate aminotransferase, ALT: alanine aminotransferase, ALP: alkaline phosphatase, $\gamma$ GTP: $\gamma$-glutamyl transpeptidase, T.bil: total bilirubin, ChE: cholineesterase, T.P.: total protain, AMY: amylase, BUN: blood urea nitrogen, CRE: creatinine, ICG: indocyanine green, AFP: alpha-fetoprotain, CEA: carcinoembryonic antigen.

From the Department of Internal Medicine, Fukuchiyama City Hospital, Kyoto Received for publication March 21, 2000; Accepted for publication July 16, 2000 Reprint requests should be adressed to Dr. Koji Inaba, the Department of Internal Medicine, Fukuchiyama City Hospital, 231 Atsunaka-cho, Fukuchiyama, Kyoto 620-8505 


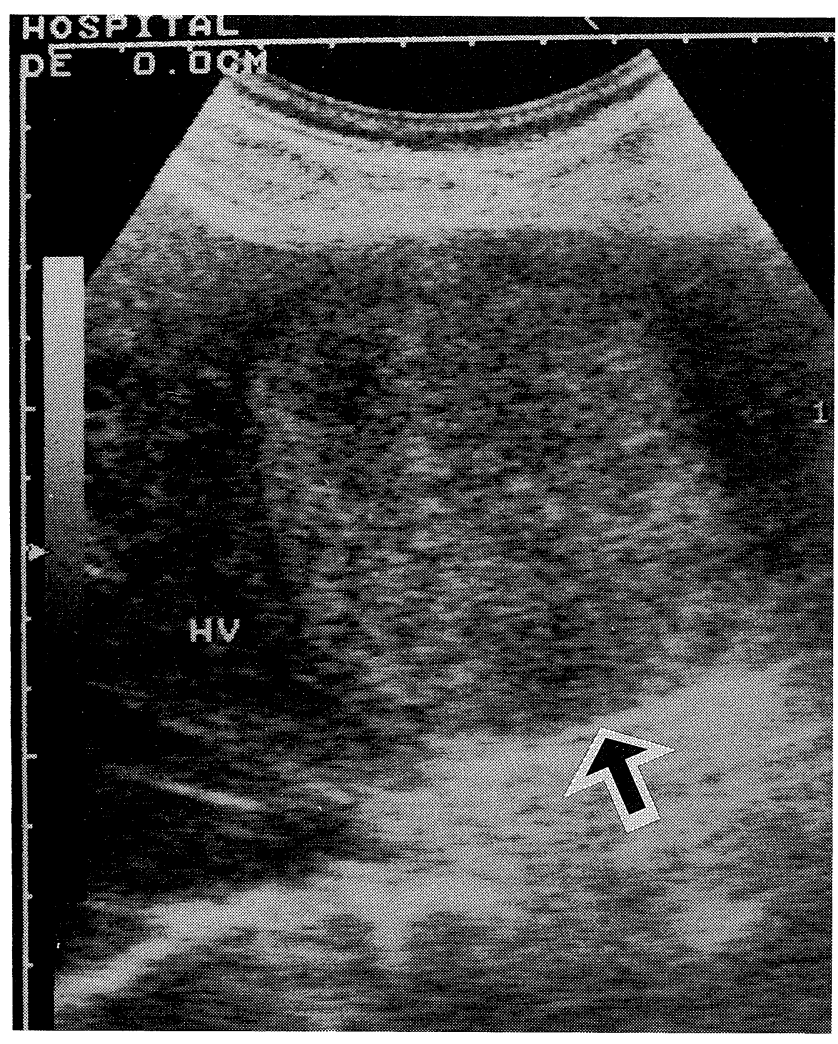

A



$\mathrm{B}$

Figure 1. A) Abdominal ultrasonography showed a hyper echoic tumor $(\rightarrow)$ at the lateral segment of the liver. B) Hyper echoic tumor $(\rightarrow)$ at the tail of pancreas.

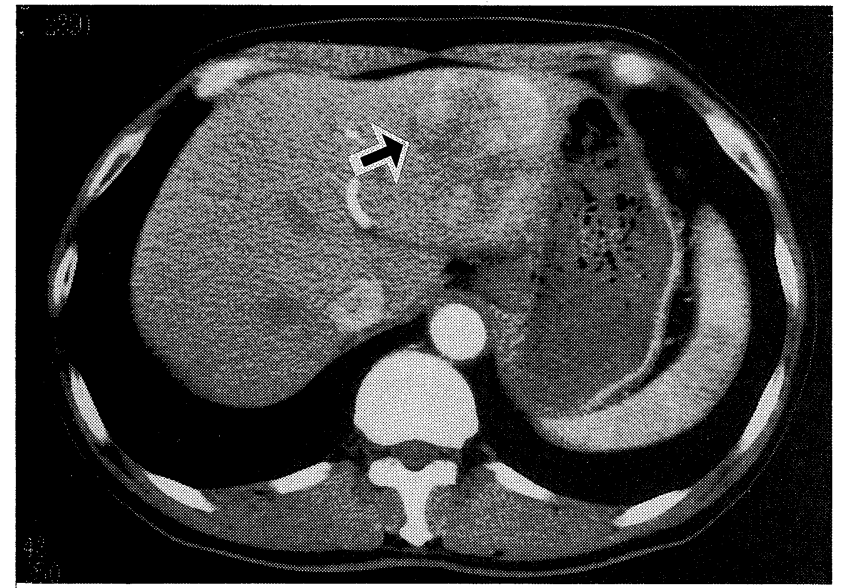

A

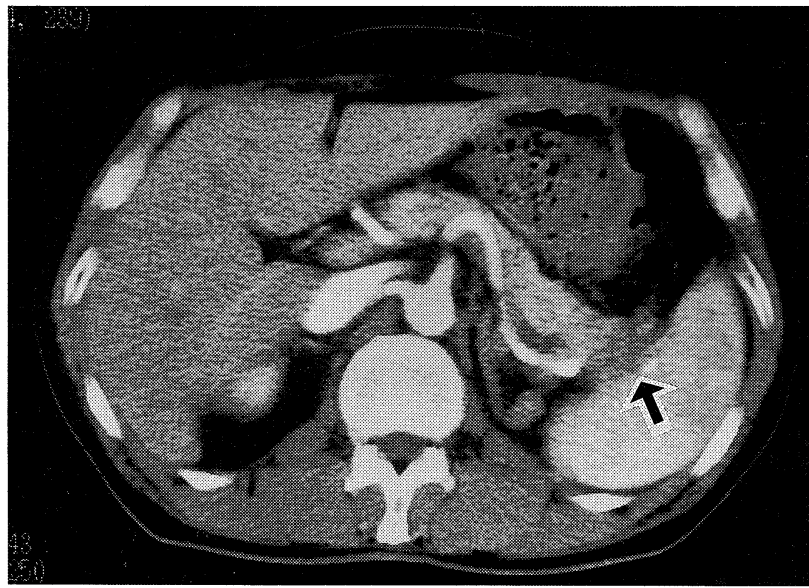

B

Figure 2. A) Dynamic contrast CT scan showed rapid enhancement effect of the hepatic tumor $(\rightarrow)$. B) The enhancement effect of the pancreatic tumor $(\rightarrow)$ was poor. 
ultrasonography revealed that a splenic vein was compressed by the pancreatic tail tumor. Endoscopic retrograde pancreatography showed discontinuance of the main duct at pancreastail. Angiography of the left hepatic artery showed hypervascular liver tumor that was rapidly and durably enhanced. Arterial-portal shunting (A-P shunt) was not recog- nized. Angiography of the dorsal pancreatic artery showed a lucent area at the tail of the pancreas. The splenic arteriogram showed encasement, and the venogram showed no blood flow of the splenic vein and collateral circulation (Fig. 4).

We considered that the pancreatic tumor was pancreatic carcinoma with invasion into the splenic vein. At first, we consid-

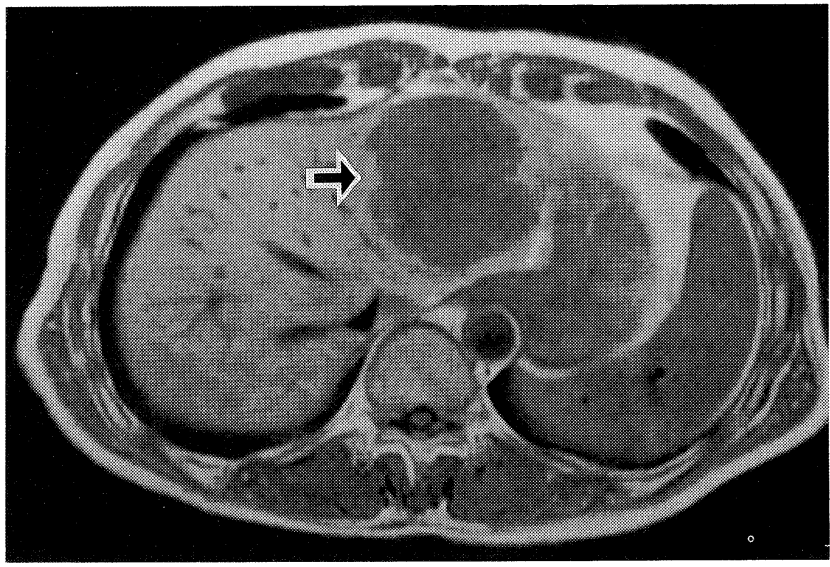

A

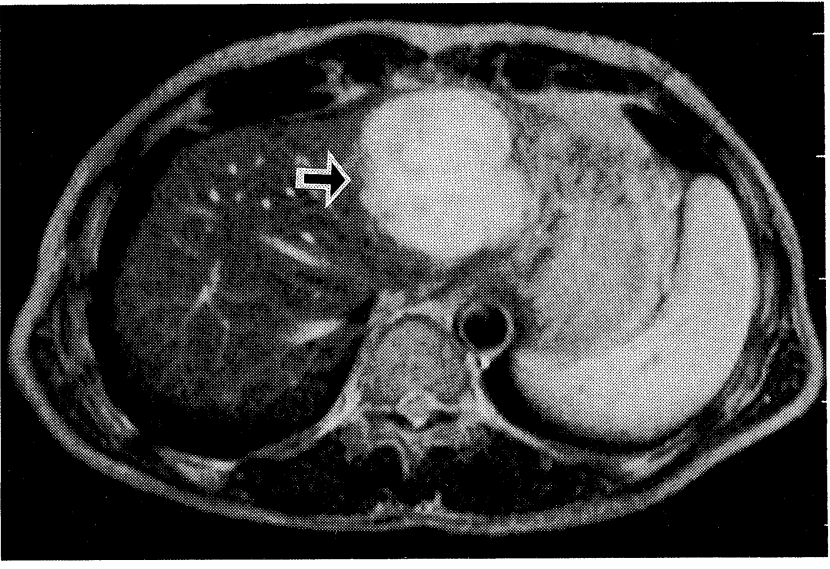

B

Figure 3. A) T1-weighted MRI showed the hepatic tumor as a low intensity mass $(\rightarrow)$. B) T2-weighted MRI showed it as avery high intensity mass $(\rightarrow)$.

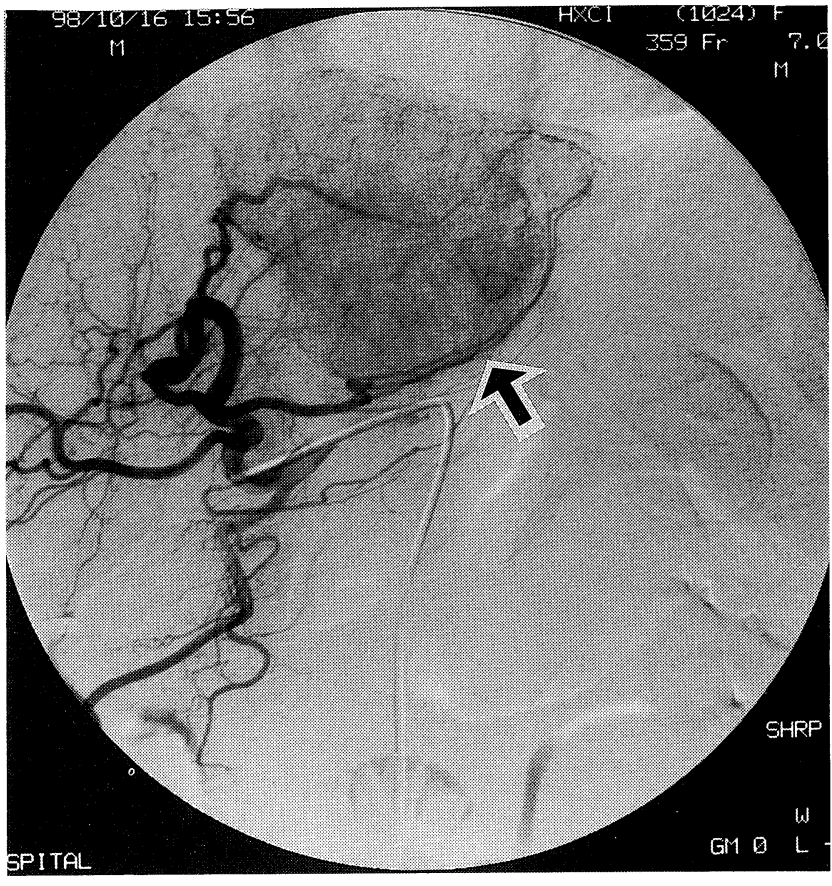

A

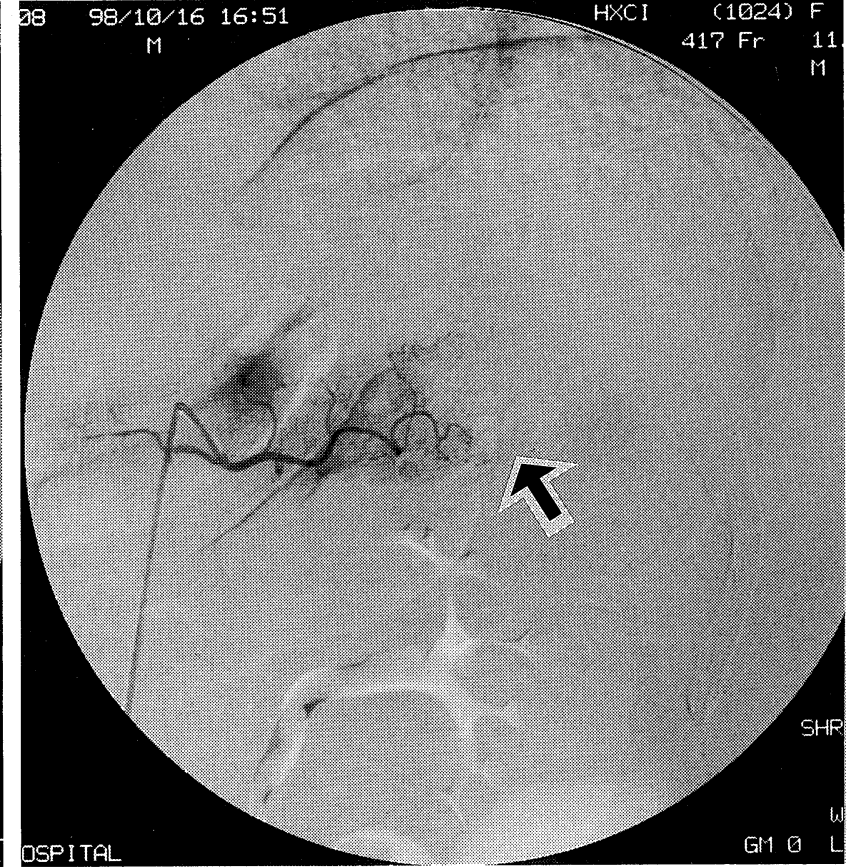

B

Figure 4. A) Angiography of the left hepatic artery showed a hypervascular liver tumor( $\rightarrow$ ). B) Angiography of the dorsal pancreatic artery showed a lucent area $(\rightarrow)$ at the tail of the pancreas. 


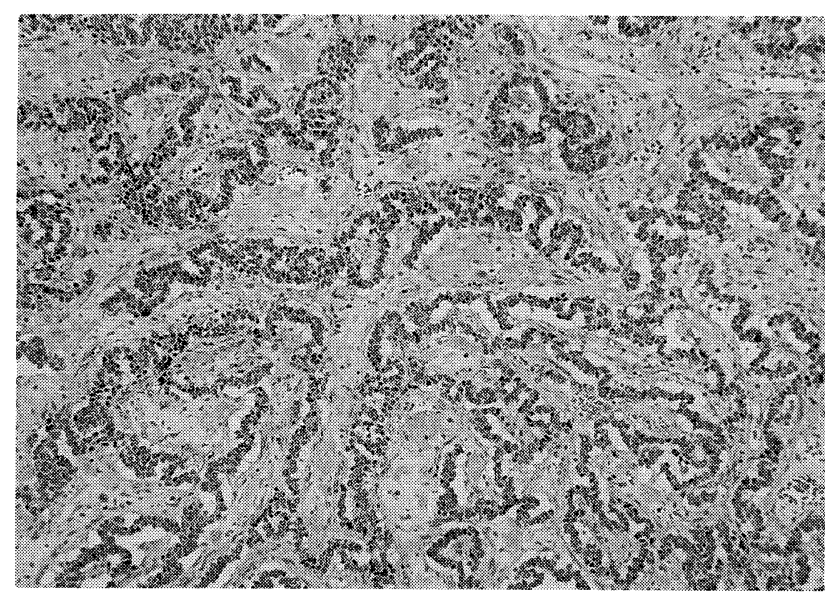

A

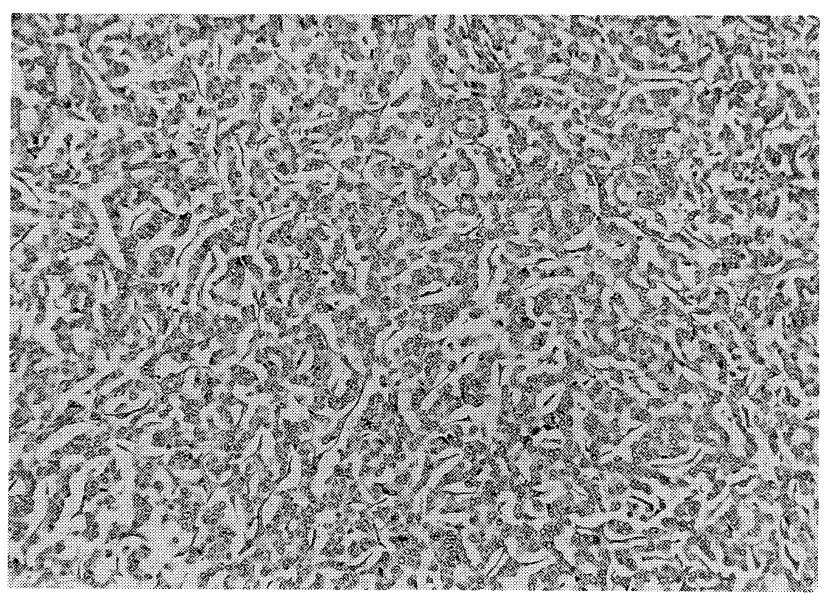

$\mathrm{B}$

Figure 5. Histology reveals ductal cell carcinoma of the pancreas (A), and metastatic liver carcinoma from the pancreas. The growth of stroma in the hepatic tumor was remarkable compared with the pancreatic tumor (B) (HE stain, $\times 40)$.

ered the hepatic tumor as cavernous hemangioma from the findings of ultrasonography and MRI. The very high intensity mass on T2-weighted MRI is characteristic of cavernous hemangioma, but hepatocellular carcinoma (HCC) or liver metastasis from a hypervascular tumor cannot be denied. Rapid enhancement effect by CT and angiography was not compatible with cavernous hemangioma. So that, we considered a hepatic tumor HCC, though the patient had no chronic liver diseases or viral markers such as $\mathrm{HBs}$ antigen and $\mathrm{HCV}$ antibody which were negative. It is impossible to necrotize huge HCC completely by transcatheter arterial embolization (TAE). On the other hand, the prognosis is poor if pancreatic cartinoma is unresectable, and surgical resection is the only potential treatment for pancreatic carcinoma. In the present case, we thought that pancreatic carcinoma was resectable even though it was advanced.

The laparotomy was done on November 2, 1998. Alarge hard tumor of the liver was revealed at S3, and multiple small tumors of about $10 \mathrm{~mm}$ in diameter were observed on the right. A hard tumor was revealed from the pancreas tail to splenic hilus. The splenic vein was completely obstructed at the splenic hilus. Distal pancreatectomy and left lateral hepatectomy were done for mass reduction.

Histological examination of the pancreatic tumor showed invasive ductall cell carcinoma, which was moderately differenteated tubullar adenocarcinoma, and that of the hepatic tumor showed metastatic carcinoma from the pancreas. Growth of the stroma in the hepatic tumor was remarkable compared with the pancreatic tumor. Obvious vessels were not indentified but many erythrocytes were detected in the hepatic tumor (Fig. 5).

\section{Discussion}

Pancreatic ductal cell carcinoma often occurs in liver metastasis (1). Because the vascularity of metastatic tumor is usually similar to that of primary focus, liver metastasis from pancreatic carcinoma is mostly hypovascular. Moreover, metastatic liver tumors typically show target or bull's eye sign on ultrasonography, and rim enhancement on CT scan. However, because the findings of liver tumor were quite different from that of pancreatic tumor, we considered it to be another lesion of the liver.

Angiogenesis is the essential factor in tumor development $(2,3)$. Even though it is a hypovascular tumor, angiogenesis must occur for its growth factor and metastasis (4-6). But it is usually limited in peripheral tumorous tissues. In the present case, tumor stain was recognized in not only peripheral but also central tumorous tissues. We thought these findings were compatible with hepatocellular carcinoma before the operation.

Eventually, histological examination of the pancreatic tumor and hepatic tumor showed that they were the same tumor. When we compared the two lesions, the tumor cells showed resemblence morphologically but the stromal volume of metastatic tumorous tissue was richer than that of the primary focus. It is suggested that the difference in the stromal volume is rerated to the difference in the vascularity. Therefore, we assessed angiogenesis by CD34 immunohistochemistry but the difference between two lesions was not significant. Several angiogenic foctors are produced by stromal cells such as fibroblasts and macrophages. Some factors such as vascular endothelial growth factor (VEGF) or thymidine phosphorylase (TP) originating in stromal cells might be involved in angiogenesis in liver metastasis (7-10). 


\section{Hypervascular Liver Metastasis}

We reported a very rare case of hypervascular liver metastasis in whom the vascularity of the primary focus was hypovascular. In the future, the precise involvement of angiogenic factors will be elucidated in molecular biology studies.

\section{References}

1) Matsuno S, Kato S, Kobari S, Sato T. Clinicopathological study on hematogenous metastasis of pancreatic cancer. Jpn J Surg 16: 406-411, 1986.

2) Folkman J. Angiogenesis in cancer, vascular, rheumatoid and other disease. Nat Med 1: 27-31, 1995.

3) Piate KH, Breier G, Risau W. Molecular mechanisms of developmental and tumor angiogenesis. Brain Pathol 4: 207-218, 1994.

4) Liotta LA, Kleinerman J, Saidal GM. Quantitative relationships of intervascular tumor cells, tumor vessels, and pulmonary metastases following tumor implantation. Cancer Res 34: 997-1004, 1974.
5) Mahadevan V, Hart IR. Metastasis and angiogenesis. Acta Oncol 29: $97-$ 103, 1990.

6) Weidner N, Folkman J, Pozza F, et al. Tumor angiogenesis: a new significant and independent prognostic indicator in early-stage breast carcinoma. J Natl Cancer Inst 84: 1875-1887, 1992 (see comments).

7) Mantovani A, Bussolino F, Introna M. Cytokine regulation of endothelial cell function: from molecular level to the bedside. Immunol Today 18: 231-240, 1997.

8) Leek RD, Lewis CE, Whitehouse R, Greenall M, Clarke J, Harris AL. Association of macrophage infiltration with angiogenesis and prognosis in invasive breast carcinoma. Cancer Res 56: 4625-4629, 1996.

9) Ferrara N, Davis-Smyth T. The biology of vascular endothelial growth factor. Endocr Rev 18: 4-25, 1997.

10) Takahashi $Y$, Bucana $C D$, Liu W, et al. Platelet-derived endothelial cell growth factor in human colon cancer angiogenesis: role of infiltrating cells. J Natl Cancer Inst 88: 1146-1151, 1996 (see comments). 